\title{
A Study of Anode-Supported Solid Oxide Fuel Cell Modeling and Optimization Using Neural Network and Multi-Armed Bandit Algorithm
}

\author{
Changhee Song ${ }^{1}$, Sanghoon Lee ${ }^{1}{ }^{\mathbb{D}}$, Bonhyun Gu ${ }^{1}$, Ikwhang Chang ${ }^{2}$, Gu Young Cho ${ }^{3}$, \\ Jong Dae Baek ${ }^{4, *}$ and Suk Won Cha ${ }^{1, *}$ \\ 1 Department of Mechanical and Aerospace Engineering, Seoul National University, 1 Gwanak-ro, Gwanak-gu, \\ Seoul 08826, Korea; zidane89@snu.ac.kr (C.S.); ynsc234@snu.ac.kr (S.L.); gbh980@snu.ac.kr (B.G.) \\ 2 Department of Mechanical and Automotive Engineering, Won-Kwang University, 460 Iksan-daero, Iksan, \\ Jeonbuk 54538, Korea; ikwhang2@wku.ac.kr \\ 3 Department of Mechanical Engineering, Dankook University, 152, Jukjeon-ro, Suji-gu, Yongin-si, \\ Gyeonggi-do 16890, Korea; guyoungcho@dankook.ac.kr \\ 4 Department of Automotive Engineering, Yeungnam University, 280 Daehak-ro, Gyeongsan, \\ Gyeongbuk 38541, Korea \\ * Correspondence: jdbaek@yu.ac.kr (J.D.B.); swcha@snu.ac.kr (S.W.C.); \\ Tel.: +82-53-810-3003 (J.D.B.); +82-2-880-1900 (S.W.C.)
}

Received: 2 March 2020; Accepted: 27 March 2020; Published: 2 April 2020

check for updates

\begin{abstract}
Anode-supported solid oxide fuel cells (SOFCs) model based on artificial neural network (ANN) and optimized design variables were modeled. The input parameters of the anode-supported SOFC model developed in this study are as follows: current density, temperature, electrolyte thickness, anode thickness, anode porosity, and cathode thickness. Voltage was estimated from the SOFC model with the input parameters. Numerical results show that the SOFC model constructed in this study can represent the actual SOFC characteristics very well. There are four design parameters to be optimized: electrolyte, anode, cathode thickness, and anode porosity. To derive the optimal combination of the design parameters, we have used a multi-armed bandit algorithm (MAB), and developed a methodology for deriving near-optimal parameter set without searching for all possible parameter sets.
\end{abstract}

Keywords: anode-supported solid oxide fuel cell; artificial neural network; optimization; multi-armed bandit algorithm

\section{Introduction}

Solid oxide fuel cells (SOFCs) have attracted a lot of attention because of their high energy conversion efficiency and many studies have been conducted to maximize the performance of SOFCs [1-6]. The performance of the SOFCs can be defined as the product of the available voltage given any current density. The available voltage is typically affected by three major losses of the SOFCs, which are activation loss, concentration loss, and ohmic loss. Thus, maximizing the performance of the SOFCs can be considered to minimize the major losses of the SOFCs. The major losses of SOFCs are affected not only by the material properties of the anode, the cathode, and the electrolyte, but also by the design properties such as thicknesses of the anode, the cathode, and the electrolyte [7-10]. Therefore, to improve the performance of the SOFCs, it is necessary to optimize the design parameters of the SOFCs.

Parameter optimization through experiment to identify the characteristics of actual SOFCs is time-consuming and cost-ineffective. Therefore, various numerical models based on an actual SOFC configuration have been built and there are two ways to develop the fuel cell model. One method is a 
data-driven approach, which constructs fuel cell models using machine learning techniques such as artificial neural network (ANN) and support vector machine (SVM). [11-14] The other method is a model-based approach, in which the fuel cell models are built from physical equations such as the Nernst equation or Butler-Volmer equation [15-17]. The data-driven approach has the advantage of providing a powerful tool that can simulate the complex and nonlinear characteristics of the fuel cells through data. However, the data-driven approach requires a lot of training data to build a valid model and it is difficult to interpret the model because it is a black-box model that is not constructed based on mathematical formulas. On the other hand, the model-based approach has the advantage that it does not require data to construct the model. However, the model-based approach has intrinsic difficulty to sufficiently simulate the characteristics of the fuel cell due to many factors that are not fully defined mathematically in the physical formulas. [11]

Many studies have been conducted to optimize the design variables of fuel cells. Of the optimization theories, genetic algorithm (GA) has been mostly used to find the optimal parameters of the fuel cells [18-21]. The GA has a mechanism to avoid local optimal, but there are many variables that need to be determined, so the result is very dependent on the variables. Therefore, the performance of the GA is different according to expertise of the person configuring it.

In this study, we built an anode-supported SOFC model through the ANN which is a representative methodology of the data-driven approach. After constructing the model that simulates the characteristics of anode-supported SOFCs, we conducted a study to optimize design parameters based on this model. We used a multi-armed bandit algorithm $(\mathrm{MAB})$ to find the optimal design parameter set based on the SOFC model. The MAB provides a framework for finding optimal choices efficiently given the various options and has a merit that robust result can be obtained because there are very few parameters to set compared to the GA. In this study, we developed a methodology for finding parameter set which is close to the optimal set without searching for all possible parameter sets by using the characteristics of the MAB.

\section{Modeling by Artificial Neural Network (ANN)}

\subsection{Modeling Configuration of SOFC}

The anode-supported SOFC model was constructed using the ANN, which can capture the complex and nonlinear relationship between the input parameters and output. The data required to construct the ANN-based SOFC model is obtained from the published data [22]. F. Zhao et al. experimentally studied the effects on anode-supported SOFCs according to the design parameters and the membrane electrode assembly (MEA) configurations are as follows: (1) Porous Ni + YSZ (Yttria Stabilized Zirconia) anode support, (2) Porous Ni + YSZ anode interlayer, (3) Dense YSZ electrolyte, (4) Porous LSM (Sr-Doped $\mathrm{LaMnO}_{3}$ ) + YSZ cathode interlayer, (5) Porous LSM cathode. The experiment was mainly conducted at a temperature of 800 degrees. Therefore, the ANN constructed from the above experimental data can simulate the anode-supported SOFC having MEA structure described above and operating at 800 degrees.

Since the goal of this study is not the optimization for single parameter, but the optimization for multiple parameters, we used data from the study conducted by F. Zhao et al. that experimentally analyzed the dependence of polarization in anode-supported SOFC on various parameters. The experiments were performed by changing the design parameters including the anode support porosity, the anode support, the cathode interlayer, and the electrolyte thickness. The polarization characteristics of the anode-supported SOFC have dependency on these four design parameters. The electrolyte thickness, which has a lot to do with ohmic resistance, has been proved to affect the electrical properties of the anode supported SOFC [23]. The fact that the microstructure of the electrodes associated with the activation loss and concentration loss have an effect on anode supported SOFC has been proved in a number of papers. The effect of microstructure for the anode, including anode thickness and porosity, on anode-supported SOFCs was demonstrated by References [24-26]. The influence of the polarization characteristics of the anode supported SOFC by the microstructure of the cathode was studied in reference [27]. 
In order to analyze the effect of the change of a parameter on the SOFC, the experiment was conducted by changing the value of only one parameter and fixing the rest of the parameters to standard values. The standard values for the parameters were follows: (1) Electrolyte thickness, $8 \mu \mathrm{m}$, (2) anode support thickness, $1 \mathrm{~mm}$, (3) anode support porosity, $48 \%$, (4) cathode interlayer thickness, $20 \mu \mathrm{m}$. The parameters were varied as follows: (1) Electrolyte thickness, ranging from 4 to $20 \mu \mathrm{m}$, (2) anode support thickness, ranging from 0.5 to $2.45 \mathrm{~mm}$, (3) anode support porosity, ranging from 32 to $76 \%$, (4) cathode interlayer thickness, ranging from 6 to $105 \mu \mathrm{m}$ [22]. The level of values for the design parameters are summarized in Table 1.

Table 1. The levels of the design parameters.

\begin{tabular}{ccccc}
\hline & Level 1 & Level 2 & Level 3 & Level 4 \\
\hline Electrolyte thickness $(\mu \mathrm{m})$ & 4.00 & 8.00 & 15.0 & 20.0 \\
Anode support thickness $(\mathrm{mm})$ & 0.50 & 1.00 & 1.45 & 2.45 \\
Anode support porosity $(\%)$ & 32.0 & 48.0 & 57.0 & 76.0 \\
Cathode interlayer thickness $(\mu \mathrm{m})$ & 6.00 & 20.0 & 56.0 & 105 \\
\hline
\end{tabular}

\subsection{SOFC Modeling Using ANN}

This section describes the methodology for creating the anode-supported SOFC model using the ANN. The ANN has been widely used in modeling the fuel cells because it can effectively map the complex and nonlinear relationship between the input parameters and the output values [28]. The detailed methodology of the ANN is shown in Reference [28]. In this study, the ANN model consists of an input layer, a hidden layer, and an output layer, as shown in Figure 1. Each layer in the ANN is composed of neurons, and each neuron has a corresponding weight. The weights in the ANN are initialized to arbitrary values, and are adjusted in a direction to minimize the cost function as training progresses.

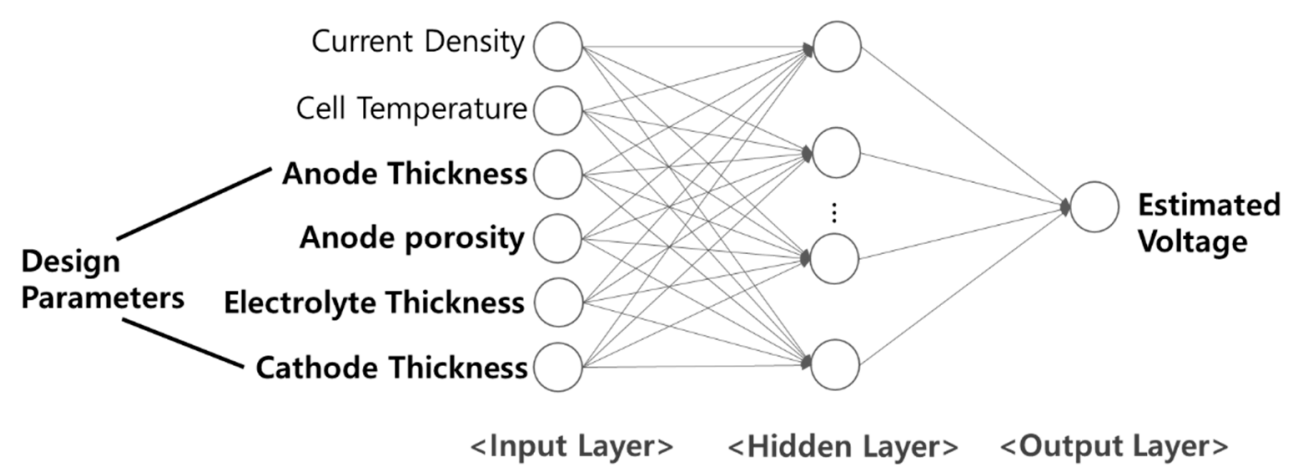

Figure 1. Artificial neural network (ANN) architecture for anode-supported solid oxide fuel cell (SOFC) model.

Six parameters entered into the input layer include temperature, current density, anode support thickness, anode support porosity, electrolyte thickness, and cathode interlayer thickness. The voltage is derived from the output layer of the ANN through calculation including a linear combination between the input parameters and weights in the ANN and a nonlinear transformation by activation functions. The structure of the ANN model used for the SOFC modeling in this study is shown in Table 2. The hyper tangent activation function is used in the hidden layer defined as Equation (1).

$$
f(z)=\frac{e^{z}-e^{-z}}{e^{z}+e^{-z}}
$$


Table 2. Information about ANN structure.

\begin{tabular}{ccc}
\hline & Number of Neurons & Activation Function \\
\hline Input layer & 6 & None \\
Hidden layer & 64 & Hyper tangent \\
Output layer & 1 & None \\
\hline
\end{tabular}

In training process of the ANN, the weights of the ANN were adjusted to minimize predefined cost functions through iterative operations based on back propagation approach. The cost function is defined by mean squared error (MSE) as shown in Equation (2) where $N, y_{i}, x$, and $\theta$ are total number of the training data, the target voltage, the input parameters, and the weights of the ANN, respectively.

$$
J=\frac{1}{N} \sum_{i=1}^{N}\left(y_{i}-f(x ; \theta)\right)^{2}
$$

The total number of data used for the SOFC modeling was 525 , and $80 \%$ of the data was used as training data for training the ANN model and the rest $20 \%$ as test data for validating the trained model. In order to prevent instability of the training process due to the numerical difference between input parameters, min-max normalization was performed so that the range of the input parameters are placed between 0 and 1. The min-max normalization is shown as Equation (3).

$$
x_{\text {norm }}=\frac{x-\min _{x} x}{\max _{x} x-\min _{x} x},
$$

Figure 2 shows a learning curve demonstrating the trend of the MSE values for the training data and the test data according to the training process. The epoch, designated as the label on the $\mathrm{x}$-axis, means the number of training for the ANN. Each epoch refers to the number of times the ANN is trained using the training data. In this study, we trained the ANN model for 200,000 epochs, which are sufficient to train. The MSE of the trained ANN model for the training and the test data are about 0.0003 and 0.0004 , respectively. Judging from that the MSE for the test data is very low as 0.0004, the trained SOFC model is in a good agreement with the actual SOFC.

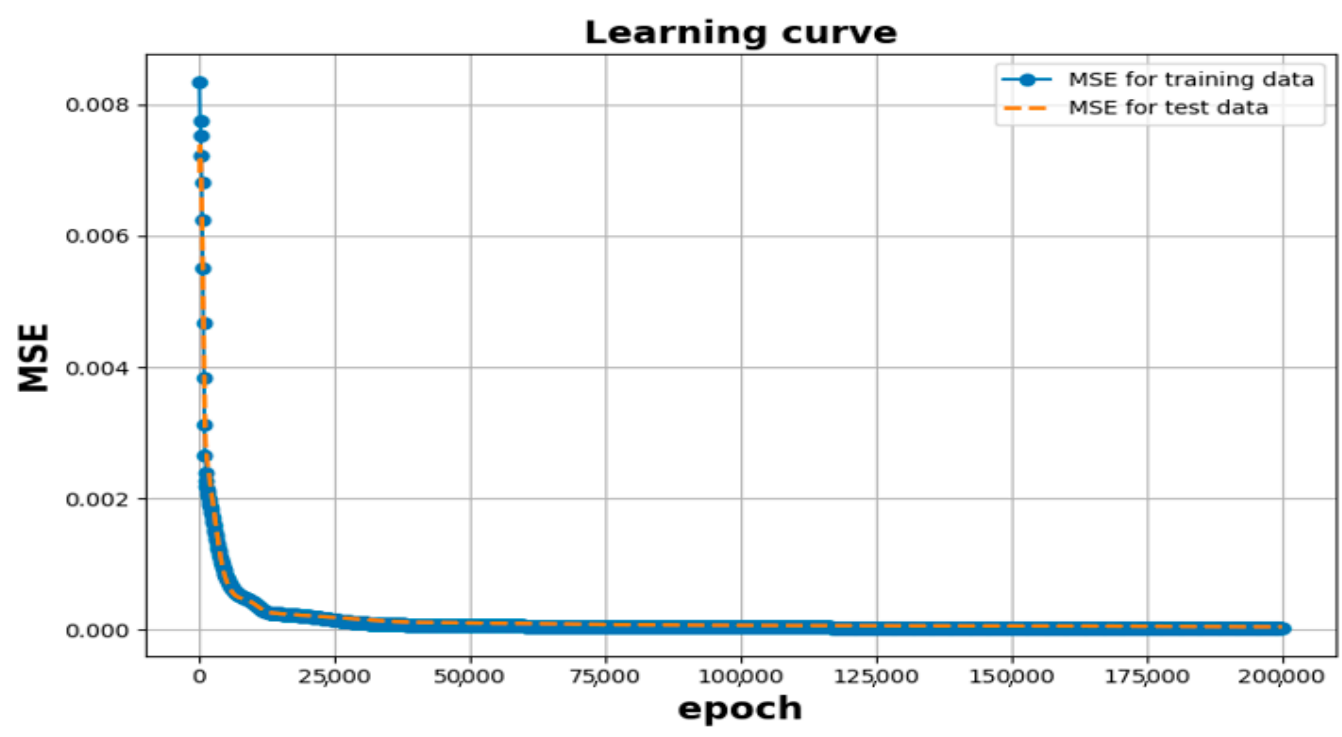

Figure 2. Learning curves for the training and the test data.

Figure 3 shows the experimental data and the polarization curves derived from the SOFC model based on the ANN according to the design parameters. The solid lines indicate the polarization 
curves derived from the SOFC model, and each point corresponds to the experimental data. We can see that the SOFC model has the ability to simulate the polarization characteristics of the actual anode-supported SOFC.

(a)

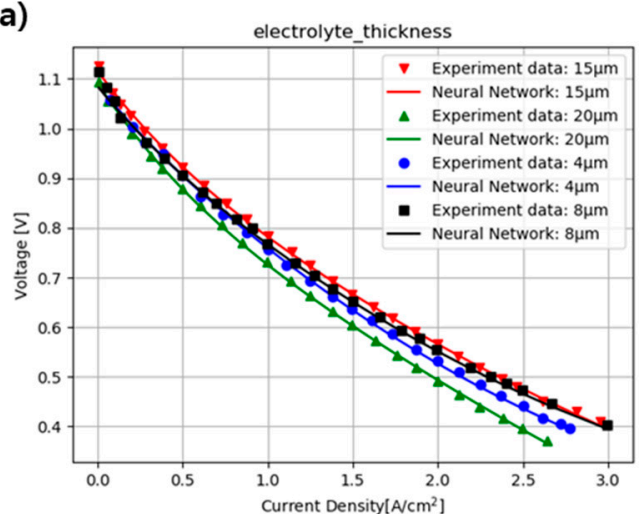

(C)

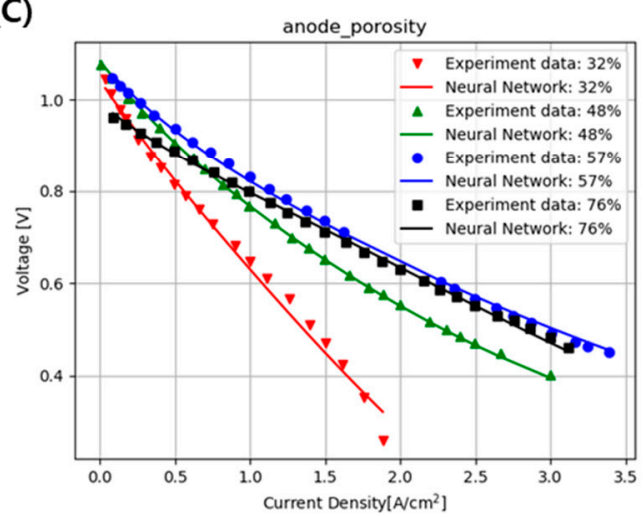

(b)

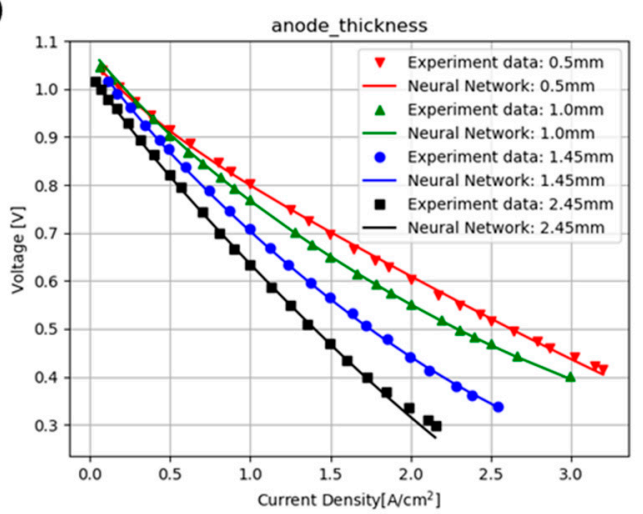

(d)

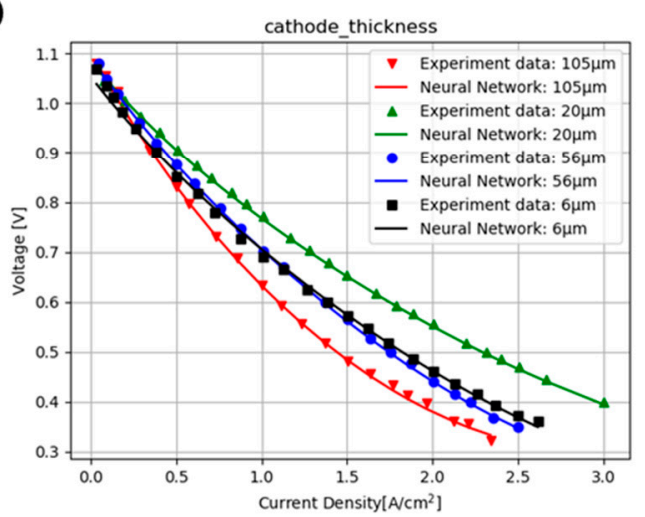

Figure 3. Experimental data points and polarization curves of the SOFC model based on the neural network for: (a) Electrolyte thickness, ranging from 4 to $20 \mu \mathrm{m}$, (b) anode support thickness, ranging from 0.5 to $2.45 \mathrm{~mm}$, (c) anode support porosity, ranging from 32 to $76 \%$, and (d) cathode interlayer thickness, ranging from 6 to $105 \mu \mathrm{m}$.

\section{Results and Discussion}

\section{Optimization of Design Parameters}

After confirming that the trained SOFC model can represent the characteristics of the actual SOFC, we conducted the optimization of the design parameters through the trained SOFC model. We searched for the optimal combination of four design parameters including anode support porosity, anode support, cathode interlayer, and electrolyte thickness. If the value of each parameter is discretized in $N$ levels, the amount of computation in brute force method which is an algorithm for searching all possible cases is $N$ to the power of 4 as in Equation (4).

$$
O(N)=N^{4}
$$

Therefore, as the number of the level values for the parameters to be optimized and the complexity of the SOFC model increase, the computation load for the optimization increases exponentially.

In this study, we introduced the multi-armed bandit algorithm (MAB) to derive the optimal combination of the design parameters efficiently. A detailed description of the MAB can be found in [29]. Figure 4 shows the schematic of the MAB. An agent in MAB chooses an action and the action is entered into the environment. The environment transfers a reward corresponding to the agent's action to the agent and the agent effectively derives the optimal action from the average value of the 
reward for multiple actions. In this work, the design parameter set corresponds to the action of the agent, and the trained ANN model corresponds to the environment.

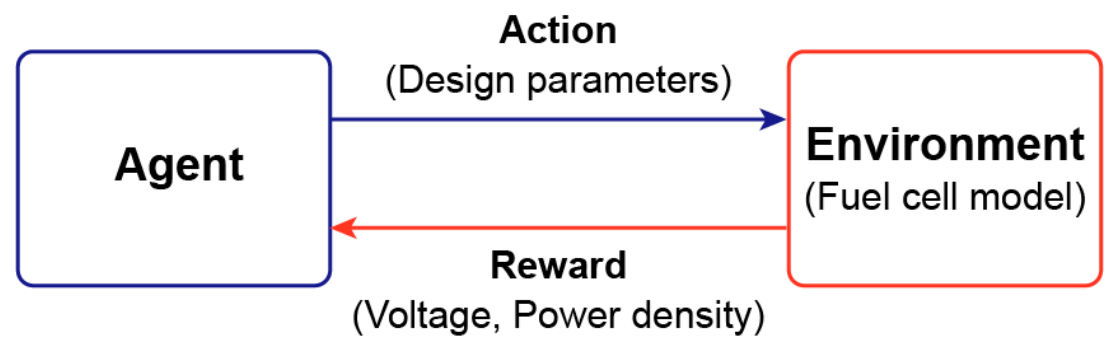

Figure 4. Schematic diagram of multi-armed bandit algorithm (MAB).

The reward was designed by considering the voltage and the power density as shown in Equation (5), where $I_{i}$ and $M$ are the current density and total number of the discretized current density, respectively, $V(x ; \theta)$ and $\gamma$ are the voltage from the ANN model and equivalent factor which equalizes power density and voltage. The current density ranging from 0.34 to $2.38 \mathrm{~A} / \mathrm{cm}^{2}$ is discretized to 10 and the equivalent factor is set to be 1 .

$$
r=\frac{1}{M} \sum_{i}^{M} I_{i} V(x ; \theta)+\gamma \cdot V(x ; \theta),
$$

We made a total of $10,000\left(10^{4}\right)$ design parameter combinations by dividing the level values of all four design parameters into 10. Therefore, in brute force method, the optimal parameter combination can be derived by 10,000 operations. In this study, the parameter set is optimized by using an epsilon-greedy algorithm ( $\varepsilon$-greedy), which is a representative algorithm of the MAB. In the $\varepsilon$-greedy, action is derived by the following Equations (6) and (7), where $Q(a), N_{t}(a)$, and 1 are an average of the reward for action ' $a$ ', the number of times the action ' $a$ ', and binary indicator factor, respectively. $\hat{a}$ and $\varepsilon$ represent an action selected randomly and the probability of selecting a random action, respectively. In this study, we set $\varepsilon$ to 0.05 .

$$
\begin{gathered}
Q(a)=\frac{1}{N_{t}(a)} \sum_{\tau=1}^{t} r_{\tau} 1\left[a_{\tau}=a\right], \\
a=\left\{\begin{array}{lr}
\arg \max _{a \in A} Q(a), & \text { with probability } 1-\varepsilon \\
\hat{a}, & \text { with probability } \varepsilon
\end{array}\right.
\end{gathered}
$$

If the optimal reward is known, regret is defined as the difference between the optimal reward and the reward for the MAB, as shown in Equation (8), and cumulative regret is the sum of the regret. In Equation (8), $r$ * represents the optimal reward.

$$
\begin{gathered}
\text { regret }=r^{*}-r \\
\text { cumulative regret }=\sum_{i}^{t} \text { regret }
\end{gathered}
$$

Figure 5a shows the cumulative regret for the $\varepsilon$-greedy and for random sampling that yields an arbitrary action. From Figure 5a, it is clearly seen that the combination of the design parameters derived from the $\varepsilon$-greedy is closer to the optimal parameter set with the simulation steps. In other words, it can be seen that the optimal solution is converged efficiently without undue computation. Figure $5 b$ shows the moving average of the relative error between the optimal reward and the actual reward according to the number of simulations. The moving average is used to reduce a noise caused by random action. 

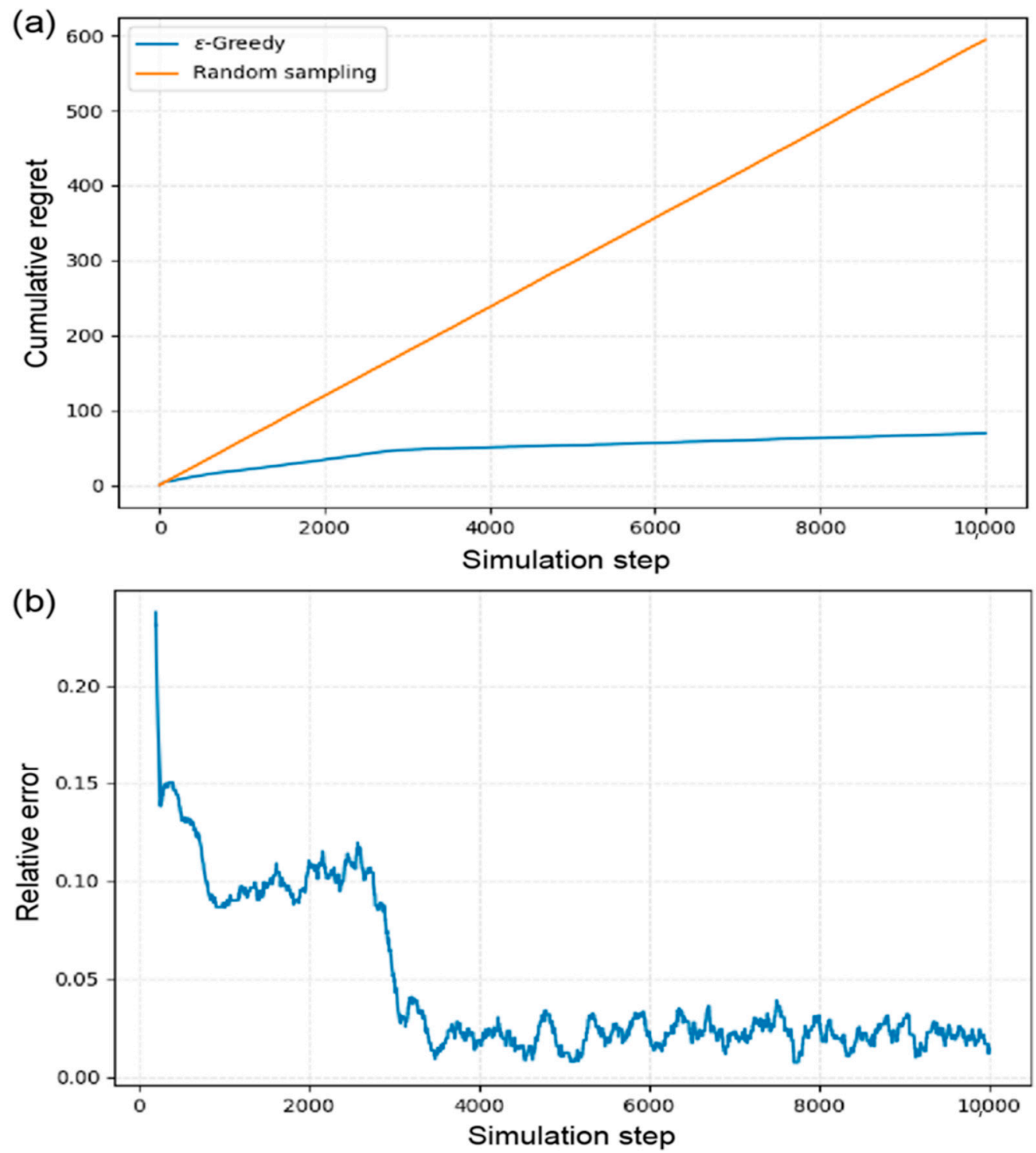

Figure 5. (a) Cumulative reward with simulation numbers. (b) Moving average of the relative error between the optimal reward and the actual reward according to the number of simulations.

Information on the design parameters derived from $\varepsilon$-greedy as the simulation progresses is shown in Table 3. Table 3 shows the selected design parameters and the reward in Equation (4) as the simulation progressed. The last column in Table $3, \Delta$ from optimal reward, represents the relative error between the optimal reward and the reward from each simulation step. The optimal reward is derived by finding the optimal design parameters through 10,000 operations using the brute-force algorithm. The polarization curves for the design parameter set selected in simulation step 500, 2000, and 4000 are shown in Figure 6. The voltage for the parameter set derived after 4000 operations is higher than the voltage for other parameter sets in all current density domains. In addition, the polarization curve for the parameter set derived after 2000 operations is generally higher than the polarization curve for the parameter set derived after 500 operations, but it can be seen that reversal of the performance occurs in the high-current region.

Figure 7 shows the distribution of the parameter values for the four design parameters in the experiment of F. Zhao et al. and the values for the optimal parameter set derived from our study. In Figure 7, the inverted triangles mean the parameter values for the study of F. Zhao et al., and the red inverted triangles indicate the parameter values showing the best performance in the experiment. In addition, the red circles mean the optimal parameter values derived from this study. Although there 
is a difference that the optimal parameters found in this study were deduced by considering all combinations of the parameters and the optimal parameters for the experiments are derived by changing the value of only one parameter and fixing the rest of the parameters to standard values, Figure 7 shows that the two optimal parameter sets show similar tendencies.

Table 3. Optimal design parameters from brute-force and MAB methods.

\begin{tabular}{ccccccc}
\hline $\begin{array}{c}\text { Simulation } \\
\text { Number }\end{array}$ & $\begin{array}{c}\text { Electrolyte } \\
\text { Thickness }(\boldsymbol{\mu m})\end{array}$ & $\begin{array}{c}\text { Anode } \\
\text { Thickness }(\mathbf{m m})\end{array}$ & $\begin{array}{c}\text { Anode } \\
\text { Porosity (\%) }\end{array}$ & $\begin{array}{c}\text { Cathode } \\
\text { Thickness }(\boldsymbol{\mu m})\end{array}$ & $\begin{array}{c}\Delta \text { from } \\
\text { Reward }\end{array}$ & $\begin{array}{c}\text { Optimal Reward }(\%) \\
\text { Optim }\end{array}$ \\
\hline 500 & 14.7 & 0.716 & 71.1 & 50.0 & 2.06 & 12.0 \\
2000 & 18.2 & 1.58 & 76.0 & 94.0 & 2.15 & 8.84 \\
4000 & 20.0 & 1.15 & 71.1 & 83.0 & 2.34 & 0.00 \\
\hline
\end{tabular}

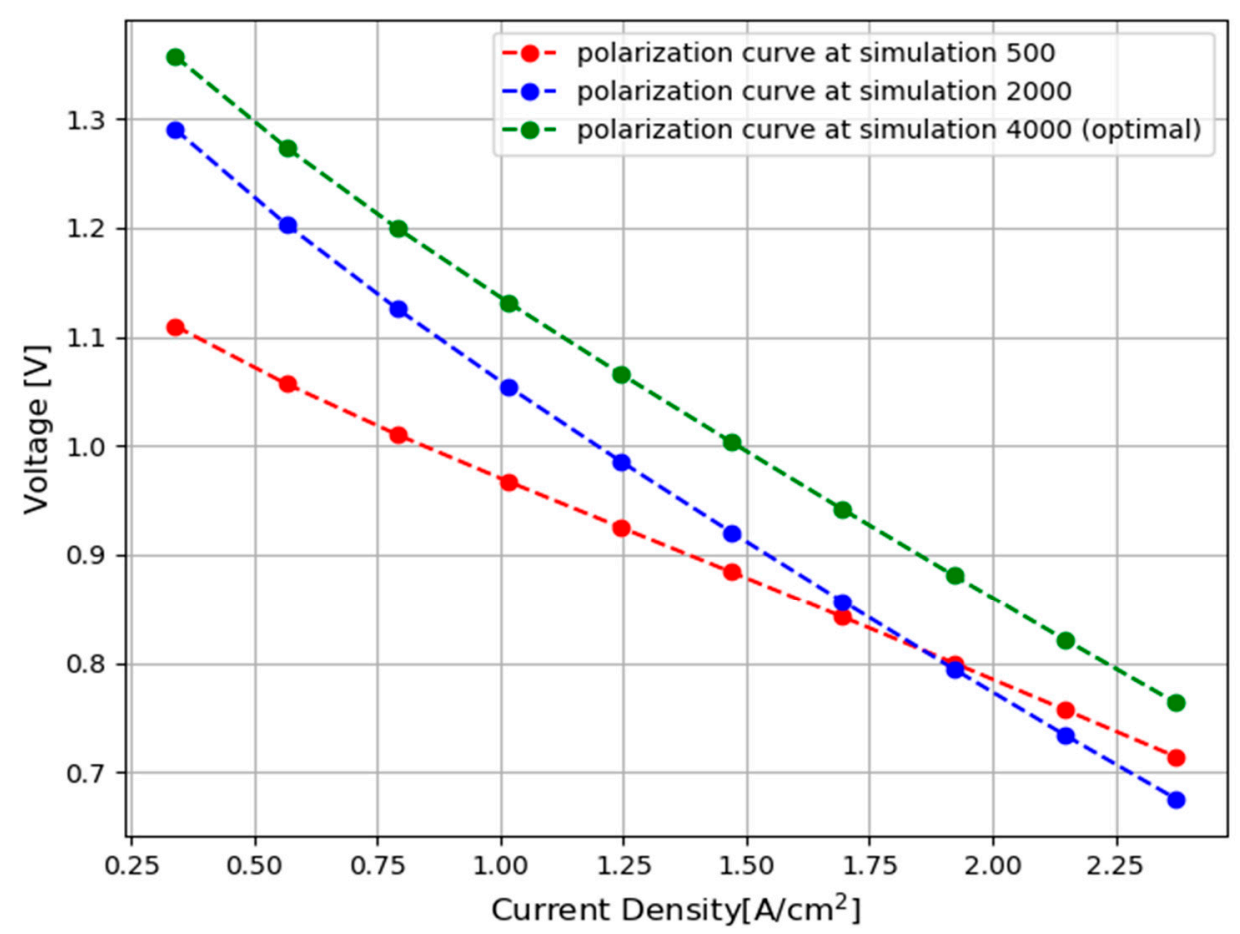

Figure 6. Polarization curves for the design parameters selected at simulation steps 500, 2000, and 4000.

Many studies have been conducted to optimize the microstructure of the anode since the microstructure of the anode support greatly affects the performance of the anode supported SOFC. Representative studies include study that analyzed the microstructure of anode in terms of thickness and porosity, conducted by $\mathrm{H}$. Noh et al. [24], and study that investigated the anode microstructure with respect to the porosity, conducted by T. Suzuki et al. [25]. In the study by H. Noh et al., the optimum anode structure has a thickness of $1 \mathrm{~mm}$, which has a thicker thickness than the comparative anode structure. It can be seen that the results of the study show a tendency similar to the optimum thickness of the anode derived from our study. Additionally, the two papers show that the large porosity of the anode improves electrical properties for the SOFC, and these results show a similar tendency to optimal anode porosity deduced from our study, which has a relatively large value.

It can be seen that the $\varepsilon$-greedy algorithm derives a parameter set in the direction of increasing reward as the simulation progresses and finds the optimal parameter through about 4000 operations. In addition, it is possible to derive the parameter set close to optimal parameter set with only 500 operations through $\varepsilon$-greedy algorithm. We confirmed that the $\varepsilon$-greedy algorithm can find parameter sets more effectively than brute-force from the simulation results. 
Electrolyte Thickness

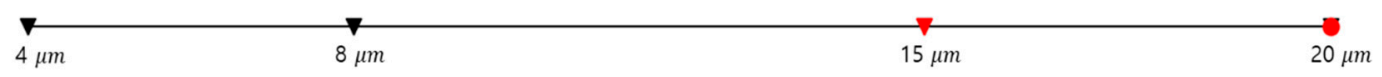

Anode Support Thickness

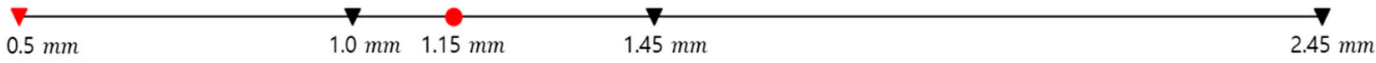

Anode Support Porosity

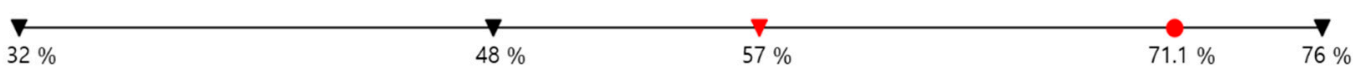

Cathode Interlayer Thickness

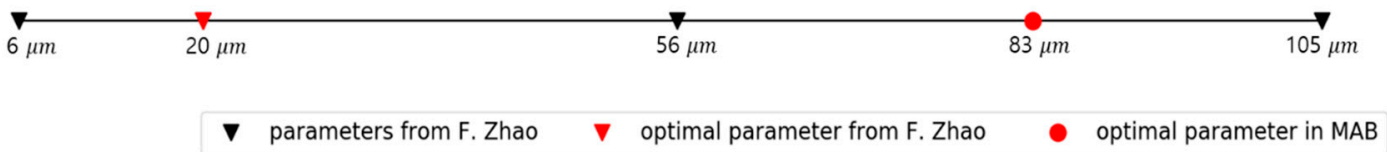

Figure 7. Comparison of the optimal parameters in our study and parameters in F. Zhao et al. study.

\section{Conclusions}

Since the performance of the SOFC is highly dependent on the design parameters, it is very important to optimize the design parameters to improve SOFC performance. In this study, the new SOFC model was presented through ANN, which can represent the complex relationship between the input parameters and the output. The presented SOFC model shows little error for the test data and simulates the characteristics of the actual SOFC well. In this study, four major design parameters of SOFC were optimized by utilizing MAB in order to derive the best combination of four design parameters. As a result, it was confirmed that the optimal set was derived with about 1/10 of the computation compared to the brute-force method used as the comparison group.

Author Contributions: C.S. established the numerical model and conducted simulations for the current manuscript with all figures and tables under supervision of J.D.B. and S.W.C. (co-corresponding authors). Additionally, S.L., B.G., and G.Y.C. gave their effort to analyze and discuss the numerical results. J.D.B., and S.W.C. as co-corresponding authors (equal contributed) and I.C., have provided useful suggestions for data interpretation and helpful comments to improve the quality of the manuscript. All authors have read and agreed to the published version of the manuscript.

Funding: This research was funded by 2018 Yeungnam University Research Grant (Grant No: 218A580052).

Acknowledgments: This research was supported by 2018 Yeungnam University Research Grant.

Conflicts of Interest: The authors declare no conflict of interest.

\section{References}

1. Chang, I.; Park, T.; Lee, J.; Lee, H.B.; Ji, S.; Lee, M.H.; Ko, S.H.; Cha, S.W. Performance enhancement in bendable fuel cell using highly conductive Ag nanowires. Int. J. Hydrogen Energy 2014, 39, 7422-7427. [CrossRef]

2. Cho, G.Y.; Lee, Y.H.; Hong, S.W.; Bae, J.; An, J.; Kim, Y.-B.; Cha, S.W. High-performance thin film solid oxide fuel cells with scandia-stabilized zirconia(ScSZ) thin film electrolyte. Int. J. Hydrogen Energy 2015, 40, 15704-15708. [CrossRef]

3. Baek, J.D.; Su, P.-C.; Yoon, Y.-J.; Lee, W.-Y. A circular membrane for nano thin film micro solid oxide fuel cells with enhanced mechanical stability. Energy Environ. Sci. 2015, 8, 3374-3380. [CrossRef] 
4. Papurello, D.; Iafrate, C.; Lanzini, A.; Santarelli, M. Trace compounds impact on SOFC performance: Experimental and modelling approach. Appl. Energy 2017, 208, 637-654. [CrossRef]

5. Fong, K.; Lee, C.K. Performance investigation of a SOFC-primed micro-combined hybrid cooling and power system in hot and humid regions. Energy 2019, 189, 189. [CrossRef]

6. Chen, G.; Luo, Y.; Sun, W.; Liu, H.; Ding, Y.; Li, Y.; Geng, S.; Yu, K.; Liu, G. Electrochemical performance of a new structured low temperature SOFC with BZY electrolyte. Int. J. Hydrogen Energy 2018, 43, 12765-12772. [CrossRef]

7. Park, T.; Chang, I.; Lee, Y.H.; Ji, S.; Cha, S.W. Analysis of operational characteristics of polymer electrolyte fuel cell with expanded graphite flow-field plates via electrochemical impedance investigation. Energy 2014, 66, 77-81. [CrossRef]

8. Cable, T.; Sofie, S. A symmetrical planar SOFC design for NASA's high specific power density requirements. J. Power Sources 2007, 174, 221-227. [CrossRef]

9. Weber, A.; Geisler, H. FEM model-based design optimization of a planar SOFC interconnector flowfield. ECS Trans. 2019, 91, 2233-2240. [CrossRef]

10. Tian, Y.; Lü, Z.; Wang, Z.; Wei, B.; Guo, X.; Wu, P. Effect of the angle between gas flow direction and electrode on single-chamber SOFC stacks. J. Solid State Electrochem. 2019, 23, 1651-1657. [CrossRef]

11. Milewski, J.; Swirski, K. Modelling the SOFC behaviours by artificial neural network. Int. J. Hydrogen Energy 2009, 34, 5546-5553. [CrossRef]

12. Wu, X.-J.; Zhu, X.-J.; Cao, G.-Y.; Tu, H.-Y. Modeling a SOFC stack based on GA-RBF neural networks identification. J. Power Sources 2007, 167, 145-150. [CrossRef]

13. Costamagna, P.; De Giorgi, A.; Moser, G.; Serpico, S.B.; Trucco, A. Data driven techniques for fault diagnosis in power generation plants based on solid oxide fuel cells. Energy Convers. Manag. 2019, 180, 281-291. [CrossRef]

14. Wu, X.; Ye, Q.; Wang, J. A hybrid prognostic model applied to SOFC prognostics. Int. J. Hydrogen Energy 2017, 42, 25008-25020. [CrossRef]

15. Bove, R.; Lunghi, P.; Msammes, N. SOFC mathematic model for systems simulations. Part one: From a micro-detailed to macro-black-box model. Int. J. Hydrogen Energy 2005, 30, 181-187. [CrossRef]

16. Recknagle, K.; Williford, R.; Chick, L.; Rector, D.; Khaleel, M. Three-dimensional thermo-fluid electrochemical modeling of planar SOFC stacks. J. Power Sources 2003, 113, 109-114. [CrossRef]

17. Tikiz, I.; Taymaz, I.; Pehlivan, H. CFD modelling and experimental validation of cell performance in a 3-D planar SOFC. Int. J. Hydrogen Energy 2019, 44, 15441-15455. [CrossRef]

18. Bozorgmehri, S.; Hamedi, M. Modeling and optimization of anode-supported solid oxide fuel cells on cell parameters via artificial neural network and genetic algorithm. Fuel Cells 2012, 1, 11-23. [CrossRef]

19. Roshandel, R.; Forough, A.B. Two strategies for multi-objective optimization of solid oxide fuel cell stacks. Int. J. Sustain. Energy 2014, 33, 854-868. [CrossRef]

20. Mukhtar, B.; Zaidi, J.S.; Faqir, N. Multi-objective function optimization for PEM fuel cell system. ECS Trans. 2010, 26, 77-88.

21. Cuneo, A.; Zaccaria, V.; Tucker, D.; Sorce, A. Gas turbine size optimization in a hybrid system considering SOFC degradation. Appl. Energy 2018, 230, 855-864. [CrossRef]

22. Zhao, F.; Virkar, A.V. Dependence of polarization in anode-supported solid oxide fuel cells on various cell parameters. J. Power Sources 2005, 141, 79-95. [CrossRef]

23. Basu, R.N.; Das Sharma, A.; Dutta, A.; Mukhopadhyay, J. Processing of high-performance anode-supported planar solid oxide fuel cell. Int. J. Hydrogen Energy 2008, 33, 5748-5754. [CrossRef]

24. Noh, H.-S.; Lee, H.; Kim, B.-K.; Lee, H.-W.; Lee, J.-H.; Son, J.-W. Microstructural factors of electrodes affecting the performance of anode-supported thin film yttria-stabilized zirconia electrolyte solid oxide fuel cells. J. Power Sources 2011, 196, 7169-7174. [CrossRef]

25. Suzuki, T.; Hasan, Z.; Funahashi, Y.; Yamaguchi, T.; Fujishiro, Y.; Awano, M. Impact of anode microstructure on solid oxide fuel cells. Science 2009, 325, 825-855. [CrossRef] [PubMed]

26. Zheng, K.; Li, L.; Ni, M. Investigation of the electrochemical active thickness of solid oxide fuel cell anode. Int. J. Hydrogen Energy 2014, 39, 12904-12912. [CrossRef]

27. Haanappel, V.A.C.; Mertens, J.; Rutenbeck, D.; Tropartz, C.; Herzhof, W.; Sebold, D.; Tietz, F. Optimisation of processing and microstructural parameters of LSM cathodes to improve the electrochemical performance of anode-supported SOFCs. J. Power Sources 2005, 141, 216-226. [CrossRef] 
28. Chen, S.; Billings, S.A. Neural networks for nonlinear dynamic system modelling and identification. Int. J. Control 1992, 56, 319-346. [CrossRef]

29. Sutton, R.; Barto, A. Reinforcement Learning: An Introduction, 2nd ed.; The MIT Press: Cambridge, MA, USA, 2018; pp. 25-42. 